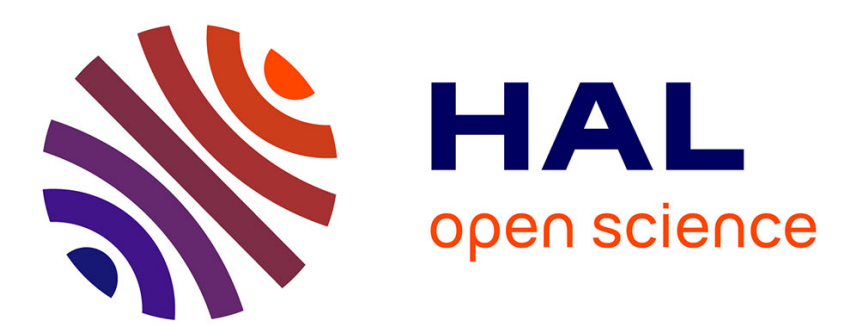

\title{
Quand les savants modernes font l'histoire des savoirs antiques : problèmes de méthode
}

Pierre Vesperini

\section{To cite this version:}

Pierre Vesperini. Quand les savants modernes font l'histoire des savoirs antiques: problèmes de méthode. Archimède: archéologie et histoire ancienne, 2019, La République " gréco-romaine " des lettres: construction des réseaux savants et circulation des savoirs dans l'Empire romain, $\mathrm{HS} \mathrm{N}^{\circ} 1$, pp.82 à 100. halshs-02091596

\section{HAL Id: halshs-02091596 \\ https://shs.hal.science/halshs-02091596}

Submitted on 5 Apr 2019

HAL is a multi-disciplinary open access archive for the deposit and dissemination of scientific research documents, whether they are published or not. The documents may come from teaching and research institutions in France or abroad, or from public or private research centers.
L'archive ouverte pluridisciplinaire HAL, est destinée au dépôt et à la diffusion de documents scientifiques de niveau recherche, publiés ou non, émanant des établissements d'enseignement et de recherche français ou étrangers, des laboratoires publics ou privés. 


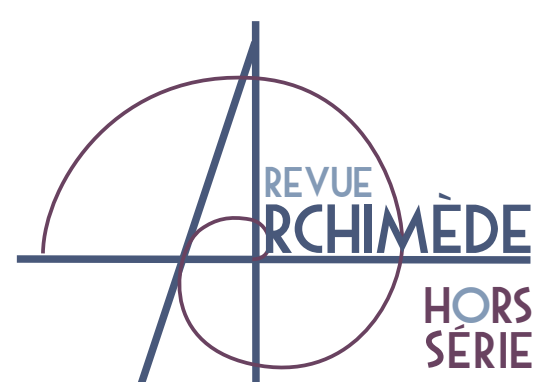

\section{QUAND LES SAVANTS MODERNES FONT L'HISTOIRE DES SAVOIRS ANTIQUES : PROBLĖMES DE MÉTHODE}

\section{Pierre VESPERINI}

Chargé de Recherche CNRS, UMR 8210 Anthropologie et Histoire des Mondes Antiques

(ANHIMA)

pierre.vesperini@cnrs.fr

\section{RÉSUMÉ}

Cet article propose une première esquisse de cartographie des catégories, et des oppositions de catégories, à partir desquelles les savants modernes abordent les savoirs anciens : savoirs/religions ; élites/ peuples ; discours/images ; communication/secret ; raison/mythes ; vérité/imagination ; âme/corps ; abstraction/concret ; sérieux/jeu ; intériorité/

\section{MotS-CLÉS}

Savoirs antiques, religions antiques éthique ancienne, art antique, philosophie antique, historiographie des savoirs, historiographie de la philosophie. extériorité. L'examen de ce premier tableau d'oppositions confronte ces catégories aux catégories indigènes du monde des savoirs antiques.
This paper aims to outline a preliminary mapping of the (opposing) categories from which modern scholars address ancient knowledge: knowledge/religions, elite/people, discourses/images, disclosure/secrecy, reason/myths, truth/imagination, soul/body, abstract/ concrete, seriousness/ play, interiority/exteriority. The examination of these opposing notions leads to a comparison between modern categories and the emic categories of ancient knowledge world.
KeYWORDS

Ancient knowledge, ancient religions, ancient ethics, ancient art, ancient philosophy, historiography of knowledge, historiography of philosophy. 
Ce texte, issu d'une conférence au séminaire «PLH-Erasme : Circulations des savoirs et structurations des réseaux savants dans I'Empire "grécoromain" », a conservé, même dans sa version écrite et révisée, la forme qui caractérise la communication orale savante. J'ai bien conscience en effet que mon sujet exige les dimensions d'un livre et non celle d'un article, encore moins d'un article issu d'une conférence. Si je choisis de proposer ce texte, donc de courir le risque du « discours délibérément imprudent »[1], c'est parce que j'espère ainsi ouvrir une discussion sur ce qu'on pourrait appeler l'imaginaire savant des savoirs antiques, la façon dont les spécialistes d'aujourd'hui se représentent, se figurent, les savoirs antiques.

De quoi parle-t-on quand on dit « circulation des savoirs », « mondes savants », « réseaux savants »? De quels savoirs parle-t-on ? De quels « savoirs » ces savants sont-ils les savants ? La plupart du temps, quand nous disons « savoirs», nous voulons dire «savoirs lettrés », ou encore « savoirs intellectuels ». Nous ne parlons pas du savoir des cuisiniers, des pantomimes, des sculpteurs, des acteurs, des devins et alii. Nous parlons de Plutarque, d'Athénée, de Pline I'Ancien et de Pline le Jeune, d'Aulu-Gelle, d'Aelius Aristide, de Philostrate, et de leurs amis. Autrement dit, nous découpons un espace dans l'espace des savoirs antiques, et nous faisons comme si cet espace correspondait à l'ensemble des savoirs. Et c'est à mon sens un premier problème. Ce problème ne peut se résoudre qu'en partant non pas de nos catégories (« savoirs », « lettré », « intellectuel »), mais en partant des catégories antiques.

Notre catégorie de «savoir » pose en effet problème : elle ne coïncide avec aucune catégorie

[1] BOURDIEU 1987, p. 10.

[2] Je fais référence ici à une épitaphe poétique bien connue des historiens de l'économie et de l'artisanat antique (CIL XII $722=$ CLE 483) : « Aux dieux Mânes de Quintus Candidius Benignus, membre du corps des charpentiers d'Arles. Le plus grand savoir (ars), la passion (studium), la culture (doctrina) et la pudeur le caractérisaient, lui que les plus grands parmi les hommes de métier (artifices) ont toujours qualifié de maître (magistrum). Personne ne fut plus savant (doctior), personne ne pouvait le surpasser, lui qui savait construire des machines hydrauliques ou diriger le flux des eaux. C'était un doux convive (conuiua dulcis) qui savait nourrir ses amis, capable d'enseigner (docilis), par nature et par éducation, et doté d'une âme

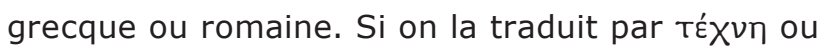
par ars, alors il faut prendre en compte les savoirs des cuisiniers, des pantomimes, des sculpteurs, des banquiers, des foulons, des constructeurs d'appareils hydrauliques [2], etc. Si on la traduit par

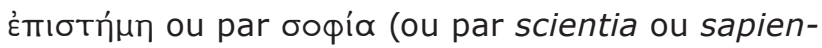
tia), alors on quitte le domaine d'Athénée, d'Aulu-Gelle, etc. Ce qui montre enfin la contingence de cette notion, c'est que nous ne pouvons même pas la traduire en anglais ou en allemand. Quand les Lieux de savoir de Christian Jacob deviennent en anglais des Places of Knowledge, on voit bien qu'on perd quelque chose. Premier point, donc : jeter le doute sur la notion de «savoir».

Intéressons-nous maintenant aux expressions « savoirs lettrés », ou encore « savoirs intellectuels », notamment quand on les emploie à propos de Plutarque, d'Athénée, de Pline I'Ancien, d'Aulu-Gelle. De quoi parlons-nous alors ? Ici, il me semble que nous décrivons un ensemble cohérent, qui est bien attesté dans la culture antique, et

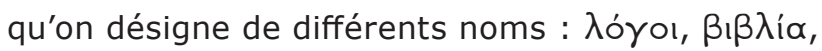

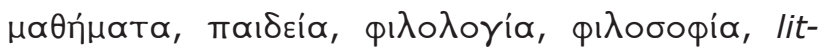
terae, doctrina, studia, artes liberales, artes (ou studia) humanitatis. Mais, cela posé, j'ajoute tout de suite qu'il faut faire attention à deux choses :

1) Il faut chercher à éviter l'usage du mot « intellectuel »: au-delà de raisons bien connues et souvent invoquées ( $y$ compris au seuil d'études portant sur... «les intellectuels dans I'Antiquité »), le principal problème de ce mot est qu'il est associé à la notion d'« abstrait », qu'il exclut immédiatement ce qui relève du « physique», du « manuel » du « concret ». Or il suffit d'ouvrir Pline I'Ancien pour voir que son Historia naturalis couvre un champ qui, pour nous, relève aussi bien

bienveillante ». Je reprends la traduction de TRAN 2013, p. 177-178, en prenant le parti de ne pas donner un sens spécifiquement artisanal à des termes qui se déploient, sur toute l'inscription, dans une indétermination générique qui rattache le charpentier au monde du savoir en général. C'est d'ailleurs ce à quoi invite Nicolas Tran (ibid.) : «Il est légitime de regarder les artisans romains comme des hommes de savoir, car c'est ainsi que se sont présentés certains d'entre eux ». Le couple ingenio/studio (qu'on rencontre souvent sous la forme ingenium/ars), le couple associant savoir et « tenue » morale, font typiquement partie de la panoplie idéale des lettrés. Noter aussi, peut-être, la notation conuiua dulcis, qui rattache peut-être le charpentier aux plaisirs des entretiens savants dans les banquets. 
de I'« intellectuel » que du «manuel ». Et quand je dis Pline I'Ancien, je parle bien sûr également de tous les «lettrés » dont il transcrit le savoir.

2) À l'inverse du mot « intellectuel », le mot «lettré » correspond bien à un mot antique : litteratus. Mais comme le disait Marc Bloch, « les hommes n'ont pas coutume, chaque fois qu'ils changent de mœurs, de changer de vocabulaire [3] » : les litterae ne sont pas nos «lettres». Elles comprennent aussi bien ce que nous appelons « lettres » (poésie, théâtre, rhétorique) que ce que nous appelons «sciences » (physique, mathématiques, géométrie, astronomie, biologie), « arts » (peinture, sculpture), « sciences humaines » (histoire, géographie), « musique », « médecine », «droit», « économie », « agronomie », ainsi que des savoirs qui pour nous ne sont pas des savoirs : physiognomonie, astrologie, oniromancie, mythologie, divination, paradoxographie.

Résumons : ce que les antiquisants appellent couramment «I'histoire des savoirs antiques » couvre un champ plus restreint, en réalité, que le champ de tout ce que les Anciens pouvaient assimiler à

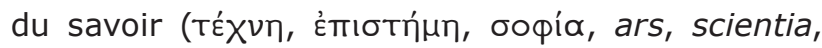
sapientia). Et ce champ plus restreint, nous le travaillons trop souvent avec des catégories « modernes » (« savoir », « intellectuel »), même quand elles semblent calquées sur des catégories antiques (« lettré »/litteratus).

Comment faire, dès lors, pour échapper à nos façons de penser ? Il faut d'abord partir des catégories des Anciens : cela veut dire les « croire » [4]. Un exemple : en matière $d$ 'histoire des savoirs antiques, un des débats régulièrement suspendus, régulièrement rouverts, concerne les différences entre «philosophie » et « rhétorique »: Apulée, par exemple, est-il un philosophe ou un rhéteur ?

[3] BLOCH 1961, p. 8.

[4] Cf. GeERTZ 1974.

[5] Cf. HAHN 1989, p. 49-50.

[6] ILAlg I 2115 : [Apuleio [P/]atonico [Mad]aurenses ciues. Cité par HAHN 1989, p. 50, n. 24.

[7] WitTGENSTEIN 1953, § 19, 23 et 241.

[8] WitTGENSTEIN 1953, § 23 : « Das Wort "Sprachspiel" soll hier hervorheben, daß das Sprechen der Sprache ein Teil ist einer Tätigkeit, oder einer Lebensform. » (« Le mot "jeu de langage" doit ici mettre en évidence le fait que parler un langage fait partie d'une activité ou encore d'une forme de vie. »)

[9] HADOT 2003, p. 160 : « [...] en fait, il y a un comportement caractéristique de chaque école. Il y aurait d'ailleurs une étude à faire, qui, à ma connaissance, n'a jamais été faite d'une manière exhaustive : comment les
Il se présente comme un philosophus dans l'Apologie. Mais, aujourd'hui, la plupart des spécialistes lui refusent ce titre, au nom d'une conception moderne de la philosophie [5]. On confond ici deux choses : ce que peut être un philosophe pour nous, ce qu'il peut être pour les Anciens. Il me semble que le rôle de l'historien doit être d'enregistrer ce que lui disent les témoignages - Apulée se présente comme un philosophus platonicus, ses concitoyens de Madaure l'honorent comme tel ( $p h i$ losophus platonicus) - et d'essayer de comprendre ce qu'ils signifient [6], c'est-à-dire les « activités», les «formes de vie», pour le dire avec le Wittgenstein des Recherches philosophiques [7], auxquelles ces témoignages se réfèrent. Pour cela, ces témoignages ne doivent pas être lus comme des « documents » transparents, qui se donneraient à lire comme des comptes rendus d'expériences ou des procès-verbaux : il faut d'abord comprendre à quels « jeux de langage »- ici aussi, je renvoie à Wittgenstein [8] - ils correspondent. On voit souvent par exemple des passages de comédies (grecques ou romaines) utilisés comme des sources sur les sociétés antiques en général, et sur les savoirs antiques en particulier, sans que soit posée la question du statut singulier, comique, de ces textes [9].

Parallèlement à cette prise en compte par I'historien de ce que disent les Anciens, il lui faut aussi objectiver ses propres façons de penser : c'est-àdire ses façons de saisir, de découper, de classer, de juger, de hiérarchiser les «figures de savoir», les «pratiques de savoir » et les « objets de savoir » que lui offre I'Antiquité. Ce que Michel Foucault, en recourant à la notion kantienne d'a priori [10], appelait l'a priori historique [11]. Il ne suffit donc pas d'objectiver ce que nous entendons par « savoir »

auteurs comiques, et donc les gens du peuple, voyaient les différentes écoles de philosophie. Ainsi, les platoniciens étaient considérés comme orgueilleux, ayant [...] "le sourcil hautain". Les épicuriens, eux, avaient la réputation de ne rien manger. [...] Les stoïciens étaient regardés comme des gens exagérément austères. Les seuls qu'on ne remarquait pas, c'était les sceptiques, parce que c'étaient des conformistes. Cela, c'est I'aspect extérieur, vu par les auteurs comiques ». Cf. aussi KORHONEN 2007.

[10] La connaissance a priori chez Kant s'oppose à la connaissance empirique. Elle est fondée sur des catégories « déjà » présentes dans notre entendement, « avant » I'expérience.

[11] FOUCAULT 1966, p. 13. Cf. aussi l'idée d'a priori sociaux qu'on trouve dans la sociologie du savoir de Max Scheler, par exemple dans SCHELER 1926, p. 47-58. Je dois cette référence à HADOT 2010, p. 76. 
aujourd'hui, mais il faut encore objectiver tout le discours qui se développe autour de cette conception moderne du savoir.

Comment s'y prendre ? Il me semble qu'une façon possible consiste à jouer à l'anthropologue avec notre propre culture : supposons qu'un anthropologue doive rendre compte de notre conception du savoir et des représentations qui lui sont associées, que dirait-il ? Peut-être dresserait-il un tableau un peu comparable à celui-ci [12] : savoirs des prêtres (égyptiens, romains, grecs...), les savoirs transmis dans les cultes à mystères

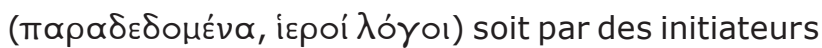
appartenant au clergé des sanctuaires à mystères (comme ceux d'Éleusis) soit par des initiateurs itinérants (mages, orphiques, « dionysiaques »[13]). Or, les cultes initiatiques sont bien liés à la question du savoir :

- par l'importance centrale de l'étape de la

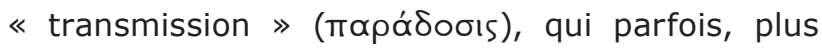

\begin{tabular}{|c|c|}
\hline savoirs & religion(s), religieux \\
\hline élites & peuple \\
\hline discours & images \\
\hline communication, diffusion & secret \\
\hline organisation (disciplines, systèmes, & absence d'organisation \\
\hline rationalité, vérité & imagination, mythes, fables \\
\hline âme, intellect, esprit & corps, physique \\
\hline abstraction & concret \\
\hline sérieux (engagement personnel, convictions) & futilité, gratuité, jeu \\
\hline intériorité & extériorité \\
\hline
\end{tabular}

1. Prenons la première ligne du tableau. À l'arrière-plan de nos discours sur les savoirs antiques, il y a souvent cette opposition, caractéristique de notre monde, entre «savoirs » et « religions ». Un savant pour nous n'est pas un religieux. Parfois même, cette distinction devient opposition : un savant, en tant que tel, devrait s'opposer au religieux.

Cela entraîne deux conséquences dans nos discours sur les savoirs antiques : nous allons a priori exclure les savoirs qui pour nous relèvent du « religieux ». Par exemple, nous ne comprendrons pas, a priori, dans les « savoirs antiques», les qu'une étape, apparaît comme l'essence même des mystères [14];

- par l'étape finale de l'illumination ('̇motreía) dans les mystères d'Éleusis, qui consiste, nous disent les sources, en une forme de connaissance visuelle, tactile, directe, foudroyante, et incommunicable ;

- par le statut paradigmatique de ces cultes aux yeux des philosophes, en particulier Platon, Aristote et Épicure. Le stade suprême de la connaissance est pensé par exemple, dans un fragment d'Aristote, par rapport à Éleusis [15].
[12] Il y a longtemps que la sémantique des oppositions n'est plus à la mode en anthropologie. Mais je crois qu'elle reste utile pour décrire les représentations à I'œuvre dans notre propre culture, étant donné son fonctionnement très souvent dualiste.

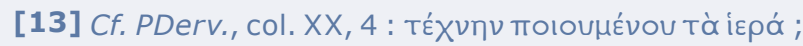

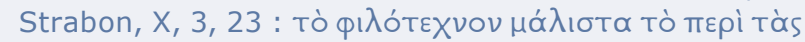

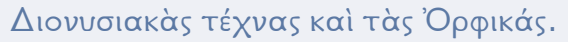

[14] Cf. Chrysippe, Stoicorum veterum fragmenta, II, 42 (p. 16-17), cité par BURKERT 1991, p. 59 : « Für den Stoiker Chrysipp ist der "logos über die Götter" das Wesen von teletai »; ou encore Athénée, II, $40 \mathrm{~d}$, qui définit les mystères comme « une fête accompagnée de

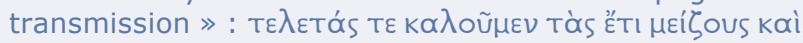

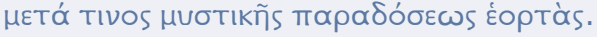

[15] Aristote, Eudème, fr. 10 (éd. Ross) = Plutarque, Isis et Osiris, 382 d-e. 
Deuxième conséquence : dans les savoirs que nous prenons en compte (les « savoirs lettrés», les savoirs des « intellectuels»), nous avons tendance à négliger, voire à « manquer», la dimension religieuse des figures, des pratiques et des objets qui sont liés à ces savoirs. Quand on parle des « savants » du « Musée » d'Alexandrie, il est rare que nous «entendions », derrière «Musée », MovбEĨov, c'est-à-dire «Temple des Muses ». Même quand la chose est rappelée, il est rare que l'historien cherche à comprendre en profondeur le sens de cette inscription d'une activité « savante » dans un espace « religieux ». Elle est pourtant omniprésente dans le monde antique. La bibliothèque fille de celle du «Musée » se trouvait également dans un temple, celui de Sérapis. Qui sait que le Péripatéticien Démétrius de Phalère, conseiller de Ptolémée, et qui fut vraisemblablement le concepteur de la bibliothèque d'Alexandrie, composa des péans en I'honneur de Sérapis, qu'on chantait encore à l'époque de Diogène Laërce [16] ?

Si nous quittons Alexandrie, véritable «métropole » de nos « savoirs lettrés», nous retrouvons ailleurs cette inscription d'une activité « savante », « intellectuelle » dans un espace religieux : par exemple dans les écoles philosophiques athéniennes [17], et jusque chez les «Ioniens », qui incarnent souvent, dans les discours modernes, les fondateurs d'une pensée et d'une science non-religieuse : où Thalès fait-il de la géométrie ? Dans le temple d'Apollon à Didymes. Que fait-il après être parvenu à inscrire un triangle dans un cercle ? Il sacrifie un bœuf. Même rapport aux dieux chez Anaxagore : comme d'autres бoфoí, Pindare ou Archiloque, il reçoit après sa mort un culte héroïque [18].

Il n'est pas difficile de comprendre les raisons de cette occultation du religieux, qui résulte des conditions dans lesquelles s'est construit le champ académique moderne, au premier rang desquelles il

[16] Diogène Laërce, V, 76 ; Artémidore, II, 39.

[17] Cf.. BoYAnCÉ 1972 ; Festugière 1968 ; KoCH PietTre 2005.

[18] Je me permets de renvoyer ici à VESPERINI 2017.

[19] À titre d'exemple, le programme 2015 du séminaire «PLH-Erasme : Circulations des savoirs et structurations des réseaux savants dans I'Empire "gréco-romain" » ne porte que sur des figures de lettrés : Nicolas de Damas, Cicéron, Athénée, Aelius Aristide, Aulu-Gelle, Sénèque.

[20] Athénée, I, $19 d$. Par exemple : «Pourquoi les éponges boivent mais ne s'enivrent pas ? ». Cf. la notice que lui consacre R. Goulet dans le Dictionnaire des philosophes antiques (M 50).

[21] Cicéron, Fin., V, 52 : quid quod homines infima faut compter l'émancipation de la tutelle de l'Église. Pour nous, a priori, un homme de savoir exerce son activité en dehors du religieux. Entre la fin du XIXe siècle et les années 1950, l'extraordinaire essor des sciences religieuses de I'Antiquité (d'ailleurs dans un esprit d'Auseinandersetzung, comme disent les Allemands, c'est-à-dire dans un esprit à la fois d'affrontement et de « dialogue critique » avec le christianisme) a conduit à mettre en valeur, par ricochet, les aspects religieux des savoirs antiques. Mais les dernières décennies ont vu au contraire une dénégation croissante de ces aspects. Les liens entre les « savoirs antiques » et les « religions antiques », si constamment noués par les savants d'autrefois, ont été rompus. Cela également peut s'expliquer par l'évolution des conditions historiques dans lesquelles vivent les chercheurs.

2. Continuons à lire le tableau, et passons à l'opposition élites/peuple. Nous trouvons souvent, dans les écrits des savants modernes, ou entre les lignes, l'idée que les «savoirs antiques »auraient été I'affaire des élites [19]. C'est là encore, me semble-t-il, un reflet d'une croyance moderne, celle de l'opposition entre «culture populaire » et « culture des élites ». J'ai été au contraire très souvent frappé, au fil de mes lectures, de constater ici et là, de façon dispersée - car nos sources nous parlent souvent des couches aristocratiques et non du «gros populas», pour le dire avec La Boétie - à quel point les foules semblent s'être passionnées pour les «savoirs lettrés ». «En Grèce et à Rome », nous dit Athénée, un forain nommé Matréas passionnait les foules en parodiant les Problèmes d'Aristote [20]. Même les ouvriers (opifices), dit M. Piso, s'intéressent à l'historia (qui signifie aussi bien ici « récit » qu'« enquêtes », « problèmes ») [21]. Quand Cicéron revient sur les raisons de son succès oratoire, il explique qu'il est dû à sa culture, car Rome est une ciuitas erudita [22], et il faut éblouir

fortuna, nulla spe rerum gerendarum, opifices denique delectantur historia ? Le contexte (défense de l'école péripatéticienne) me semble indiquer qu'ici historia a le sens de « recherche », d'« enquête », comme dans L'Histoire des animaux d'Aristote ou dans ce passage des Tusculanes (I, 108) : permulta alia colligit Chrysippus, ut est in omni historia curiosus.

[22] Cf. Cicéron, Brut., 265 (C. Valerius Triarius); 321-322 (Cicéron) : nam cum propter assiduitatem in causis et industriam tum propter exquisitius et minime vulgare orationis genus animos hominum ad me dicendi nouitate conuerteram [...] nemo erat qui videretur exquisitius quam vulgus studuisse litteris, quibus fons perfectae eloquentiae continetur ; nemo qui philosophiam complexus esset, matrem omnium bene factorum beneque dictorum [...]. 
les Romains par son savoir comme les édiles par les bêtes exotiques [23]. On pourrait multiplier les exemples : les foules se passionnaient pour le savoir : elles le découvraient dans les conférences

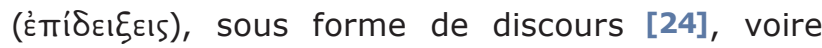
de dissections [25], mais aussi dans les livres.

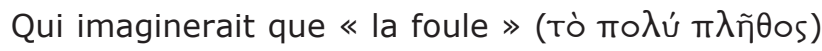
s'arrachait les livres de Plotin ? C'est pourtant ce que nous dit Eunape de Sardes [26].

Cette participation des foules au monde du savoir est confirmée par l'archéologie : à Pompéi, dans les maisons les plus modestes (appartenant à des marchands de vin ou des foulons), on rencontre des images savantes : des philosophes, Vénus Anadyomène, le célèbre pseudo-Sénèque, des mythes peints sur les murs, Dionysos, Isis, des satyres, etc. [27] Elle concorde avec une tendance récente de I'histoire de l'artisanat romain, montrant que les artisans revendiquaient le statut d'hommes savants (docti, humani) : il s'agit autant de «fierté professionnelle » que de montrer son appartenance à l'élite du monde civilisé, c'est-àdire hellénisé [28].

En dehors de cet intérêt des foules pour les savoirs lettrés, on oublie parfois que beaucoup des professionnels de ces savoirs appartenaient eux-mêmes à ces homines infima fortuna dont parle Pison dans le De finibus. Et il ne s'agit pas seulement des obscurs. Le vieux grammairien Orbilius, qui jouissait d'une excellente réputation (fama) et obtint même après sa mort une statue, se plaignait de vivre « sous les tuiles » (sub tegulis) [29]. Quand nous parlons des « intellectuels » antiques, il faut toujours distinguer entre les notables, ceux qui sont indépendants économiquement, et ceux qui doivent gagner leur vie. On ne peut pas confondre Plutarque et son concitoyen Nigros, disciple d'un « fameux philosophe » stoïcien [30], qui, pour ne pas déclarer forfait dans une joute de discours (« horrifié à l'idée

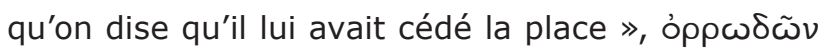

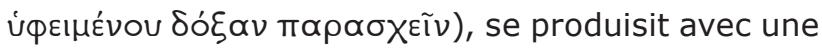
arête coincée dans la gorge, ce qui déclencha une inflammation dont il mourut (ces joutes étaient de véritables performances vocales) [31].

Les « intellectuels » qui devaient gagner leur vie philosophes, rhéteurs, grammairiens, etc. - étaient de véritables « artisans » : ce sont des vendeurs de discours [32]. On trouve dans Lucien I'histoire amusante d'un oncle qui refuse de payer pour les $\lambda$ ó $\gamma$ ol (qu'il qualifie dédaigneusement de ṕnuórtı) que le philosophe a transmis à son neveu, parce que le philosophe possède encore ce qu'il a vendu [33]. Pas plus qu'aujourd'hui, l'idée de vendre du savoir n'allait de soi. Déjà le savoir était censé être désintéressé, et émaner d'un esprit affranchi des nécessités matérielles. Je ne peux que renvoyer ici aux analyses de Pierre Bourdieu sur «I'illusion scolastique » [34]. C'est sans doute l'une des raisons pour lesquelles I'histoire des savoirs antiques est un domaine bien distinct de celui de I'histoire de l'artisanat antique. Je suis convaincu que nous aurions pourtant le plus grand profit à tirer de ces recherches : car, d'un point de vue sociologique, la plupart des « intellectuels » étaient des artisans.

3. Je serai beaucoup plus rapide sur la troisième ligne, en raison de mon ignorance. Je veux simplement dire que, a priori, nous associons le savoir à des discours et non à des images, alors que celles-ci pouvaient tout autant être chargées de savoir que les discours [35]. Cela, là encore, me semble résulter du monde dans lequel nous vivons, et non pas du monde antique. Outre la disparition
[23] Ainsi que I'explique Crassus à C. Iulius Caesar Strabo Vopiscus dans le de oratore (III, 24, 92) : Apparatu nobis opus est et rebus exquisitis, undique conlectis, arcessitis, comportatis, ut tibi, Caesar, faciendum est ad annum ; ut ego in aedilitate laboraui, quod cotidianis et vernaculis rebus satis facere me posse huic populo non putabam («Ce qu'il nous faut, à nous, c'est un cortège splendide, des raretés, collectées, recherchées, importées de partout. C'est ce que toi, César, tu devras faire I'an prochain ; comme moi je me suis escrimé pendant mon édilité : je savais qu'il était impossible de satisfaire notre peuple avec des choses qu'il voit tous les jours chez lui. »).

[24] Cf. par exemple ce que Philodème, citant la Chronologie d'Apollodore, dit du philosophe Charmadas (Ind. Acad. Herc., col. XXXII, 2-3) : grâce à sa mémoire

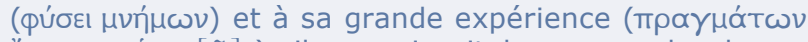

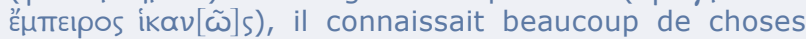

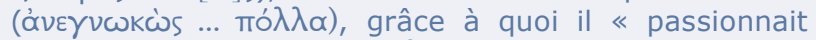

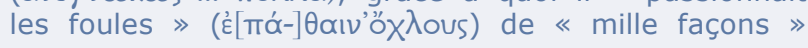

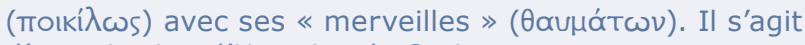
d'une citation d'Hermias de Curium.

[25] BYL 1997, p. 119-120.

[26] Eunape de Sardes, Vies des philosophes et des sophistes, III.

[27] Cf. Dessales 2017.

[28] TRAN 2013.

[29] Suétone, Gram., 9, 1.

[30] Quaest. Conv., VI, 7. On y a reconnu Épictète, cf. Cuvigny 1968, p. 565-566, suivi par PUECH 2005.

[31] Plutarque, De tuend. san., $131 a-b$.

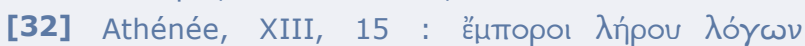
úTाokpıтñpєs. Il s'agit d'une citation d'Hermias de Curium. [33] Herm., 81.

[34] On en trouvera une bonne présentation dans BOUVERESSE 2012.

[35] Cf. la magnifique somme de LANG 2012. 
d'une immense partie des images antiques (peintures, tableaux, statues, reliefs, pièces ciselées), en comparaison avec les textes, deux facteurs au moins concourent à négliger les images : la valorisation chrétienne et postchrétienne du Livre, du Verbe, d'une Vérité qui se donne dans des formules discursives (pensez à la place, dans l'enseignement secondaire, du professeur de dessin par rapport au professeur de lettres) ; la valorisation corollaire des textes antiques, au détriment des images antiques, dans les sciences de l'Antiquité : alors qu'il est inconcevable qu'un antiquisant ignore les textes antiques, il est tout à fait concevable, et courant, qu'un antiquisant n'ait aucune familiarité avec les images antiques.

Cette hiérarchie moderne, situant les savoirs discursifs au-dessus des savoirs iconiques, n'existait pas dans l'Antiquité. Si nous possédions les écrits qui forment par exemple les sources des livres de Pline l'Ancien consacrés à la peinture, à la sculpture et à la toreutique, nous aurions peut-être une image très différente du monde des savoirs antiques.

Mais vient maintenant la question de l'interprétation de ce qu'on pourrait appeler les « images savantes ».

Il existe une approche formaliste : elle vous expliquera que le mouvement de la chevelure sur cette statuette trouvée à Pompéi se retrouve dans tel relief de l'autel de Pergame. Ces rapprochements révèlent une connaissance du corpus iconographique impressionnante. Mais en même temps, ils ne répondent pas à la question de savoir ce que signifie cette statuette, c'est-à-dire ce qu'elle signifiait dans la situation qui était la sienne pour ses différents spectateurs ou usagers.

Dans tout ce que j'ai lu à propos du sens des « images savantes », je n'ai pas trouvé de réponse vraiment satisfaisante. Ainsi, pourquoi des mythes sont-ils représentés sur les sarcophages ? Les uns proposent d'y voir des allégories religieuses ou philosophiques, les autres l'expression des sentiments du défunt face à la mort. Ces explications sont si manifestement inspirées de notre propre sensibilité qu'elles me sont suspectes. Sont-elles plus convaincantes quand on y implique la psychologie moderne? Pour rendre compte de la représentation, étonnante pour nous, du mythe d'Achille et Penthésilée sur le sarcophage d'un couple, ou celle du mythe de Phèdre et d'Hippolyte sur le sarcophage d'un fils et de sa mère, est-il utile de suggérer que les Anciens en faisaient une « lecture sélective » [36] ? Aucun témoignage antique ne vient confirmer une telle interprétation. Il en va de même pour les images que nous voyons sur les murs des maisons et des villas. J'avoue que je ne sais pas ce que signifient les fresques de la villa des Mystères, pas plus que je ne sais ce que signifie la mosaïque d'Aiôn à Antioche. Mais là aussi, l'idée que ces images exprimeraient un «message » me paraît arbitraire : comme si l'image ne «tenait » pas toute seule, sans un discours « derrière elle», pour l'étayer. De même, la catégorie de « programme » que l'on rencontre couramment pour interpréter les images, par exemple, de la villa des Papyrus à Herculanum, de I'hypogée de la Porte majeure, ou encore de la villa dite de la Farnesina à Rome, n'est pas à proprement parler une notion antique : elle remonte aux humanistes de la Renaissance (invenzione) [37]. Il y a donc là un chantier qui s'offre aux historiens des savoirs antiques : essayer de reconstituer, pour paraphraser Wittgenstein, les « jeux d'images » des Anciens.

4. Une autre idée que nous associons volontiers au savoir, c'est celle de sa diffusion, de sa communication, de sa circulation. C'est un élément essentiel de notre imaginaire savant, dont la généalogie remonte aux Lumières et, au-delà, à l'idée de République des Lettres. Dans le monde antique, la connaissance n'était pas forcément «transmissible ». Ce qui était transmissible, c'était la connaissance de type artisanal : celle que le professionnel pouvait transmettre à son apprenti. Mais on rencontre souvent l'idée que la connaissance la plus haute, la connaissance par excellence, n'est pas transmissible, car elle ne se donne pas dans du discours, mais dans une expérience comparable à celle
[36] Pucci 2014, p. 375 : «In un sarcofago le due figure centrali [...] sono chiaramente ispirate a un famoso gruppo di età ellenistica che rappresentava Achille e Pentesilea [...]. Doveva I'osservatore dedurne che il marito sepolto in quel sarcofago fosse l'assassino della moglie? Certamente no. Egli avrà piuttosto pensato a un compagno che sta accanto alla moglie nell'ora della morte e che fino all'ultimo le manifesta i suo amore. Per decorare il sarcofago di un figlio e di sua madre fu scelto il mito di Fedra e Ippolito. La scelta è quanto meno imbarazzante, dato che l'amore di Fedra per Ippolito non è un casto sentimento materno, bensì una passione incestuosa che porta entrambi alla rovina. Dobbiamo accusare di mancanza di buon gusto committenti ed esecutori? No, perché come nel caso di Achille e Pentesilea I'osservatore non richiamava alla memoria I'intero mito. L'aspetto riprovevole della vicenda di Fedra veniva messo da parte. Restava solo il grande amore per il figlio e il grande dolore per la sua perdita ». Je souligne.

[37] Je me permets de renvoyer ici à VESPERINI 2010 et VESPERINI 2015a. 
de l'ह̇тотт⿻ía [38]. Le scepticisme antique, plus qu'un courant philosophique parmi d'autres, doit être compris comme l'expression d'une représentation profondément ancrée dans le monde antique : les mortels n'ont pas accès à la connaissance, à la vérité : même s'il y avait du vrai, dit Gorgias, cité par Sextus Empiricus, on ne pourrait pas le communiquer [39]. Seuls les dieux la connaissent. Même idée dans la lettre VII de Platon : « S'il m'avait semblé qu'on pouvait écrire passablement, qu'on pouvait les formuler devant tout le monde, qu'aurais-je pu faire de plus grand dans ma vie que d'écrire <des livres> qui seraient d'une <si> grande utilité pour les hommes et porter à la lumière, devant tous, la nature <de l'univers> ? » [40].

Parallèlement à cette idée selon laquelle on ne peut transmettre que des savoirs de type artisanal, et non pas les connaissances ou les vérités les plus hautes, une autre façon de penser antique nous échappe souvent : c'est celle selon laquelle le savoir en tant que tel est beau, et doit par conséquent donner lieu à des objets esthétiques. En travaillant sur Lucrèce, j'ai été amené forcément à travailler sur la notion de «poésie didactique », et je me suis aperçu que ce genre n'existait pas. L'idée qu'on communique du savoir grâce aux charmes de la poésie est une idée qui date du XVIII ${ }^{\mathrm{e}}$ siècle. Pour les Anciens, Nicandre, Aratos et Lucrèce faisaient des épopées, dont la matière était différente des épopées d'Homère et d'Apollonios. Mais cette différence de matière n'impliquait pas une différence générique ou pragmatique [41].

5. Dans notre monde, les savoirs lettrés sont une chose relativement organisée : il y a d'abord des disciplines. À l'intérieur de ces disciplines, il y a des sous-disciplines, organisées par des catégories comme la nation (« littérature anglaise », « italienne », « russe »), le genre (« poésie », « théâtre », « roman »), la période, et à l'intérieur de ces sous-disciplines il y a des spécialités, organisées par « auteurs », par « thèmes », par « courants », « groupes », « écoles » (ce sont les

[38] VESPERINI $2017 \mathrm{~b}$.

[39] Gorgias, fr. 3 Diels-Kranz (= Sextus Empiricus, Adv. math., VII, 65) : «Dans l'écrit intitulé Sur le non étant ou Sur la nature il démontre trois principes l'un après l'autre : premièrement, rien n'est ; deuxièmement, même s'il est, il est insaisissable à l'homme ; troisièmement, même s'il est saisissable, il est incommunicable et intraduisible pour autrui ».

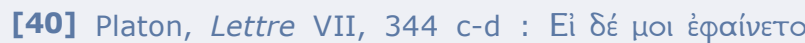

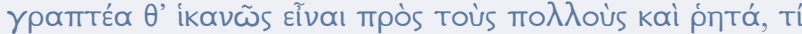

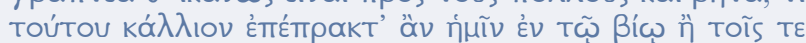

différents «-ismes »). Toute cette organisation a évidemment, là aussi, des conséquences sur notre approche des «savoirs antiques ».

\section{GENRES}

Nous cherchons souvent à identifier les critères définissant des « genres», et nous recourons parfois pour cela à des « thématiques ». Or - comme le montre par exemple très bien un livre collectif récent sur le dithyrambe [42] - les formes $(\varepsilon ` \delta \eta)$ « littéraires » des Anciens ne sont pas des genres, et les Anciens ne recourent pas, à ma connaissance, à la notion de «thématique » pour définir leurs «formes ». Un ÉTros, c'est un poème en hexamètres. Peut-on aller un peu plus loin ? C'est la forme poétique la plus élevée : c'est le « grand genre », celui qui est censé transfigurer son objet. Mais on ne peut pas aller beaucoup plus loin. Qu'estce qui rapproche Homère et Hésiode, I'Hécalè de Callimaque (rappelons au passage que le genre de

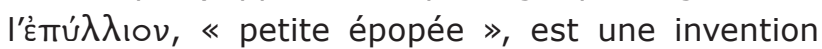
des savants modernes) et les Argonautiques ? Il faut encore compter avec les formes littéraires qui jouent avec l'épopée en recourant elles aussi aux hexamètres, mais sans la magniloquentia épique : Eidullia de Théocrite, Satirae de Lucilius, Bucoliques de Virgile. Je crois que, si l'on tentait d'établir la taxinomie des genres antiques, on obtiendrait quelque chose qui ressemblerait à l'Encyclopédie chinoise de Borges, rendue si célèbre par Foucault.

\section{NATIONS}

Ensuite, nous avons souvent tendance à envisager les savoirs en termes « nationaux » : il y aurait les savoirs égyptiens, perses, grecs, étrusques, romains, juifs, etc. Cela tient bien sûr à notre formation classique : nous sommes initiés à une Antiquité qui ignore ce que nous appelons «l'Orient » [43].

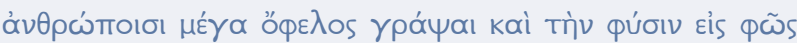

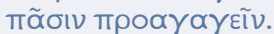

[41] Je me permets de renvoyer à VESPERINI 2015b. et Vesperini 2017 c.

[42] KOWALZIG \& WILSON 2013.

[43] En 1922, Edmond Pottier croyait pouvoir prédire que l'helléniste de l'avenir serait un orientaliste « sous peine d'ignorer la moitié de son métier ». Thierry Petit, qui le cite dans son livre remarquable sur le motif iconographique du sphinx dans les mondes chypriote, égyptien et grec (PETIT 2011, p. 115), a raison de conclure : «Nous sommes loin du compte». 
De sorte qu'ensuite, dans nos recherches, nous percevons tout savoir que nous appréhendons comme « oriental » comme un savoir d'ailleurs, exotique, sans penser que ce savoir d'ailleurs était peut-être là depuis des siècles.

C'était un monde où les communications étaient permanentes depuis l'époque archaïque : le pharaon Néchao envoyait des offrandes au temple d'Apollon à Didymes [44], comme les Athéniens allaient consulter l'oracle d'Ammon à Siwa [45]. La présence de savoirs égyptiens n'implique donc pas forcément une source égyptienne : les arétalogies d'Isis trouvées à Délos ne sont pas forcément l'expression de la propagande du clergé de Memphis [46]. Cléa, I'amie de Plutarque qui présidait au culte d'Isis à Chéronée (où le culte était installé depuis le II siècle avant notre ère) n'était pas égyptienne, pas plus que les artisans qui, à Délos, fabriquaient avec une connaissance profonde des rites égyptiens, des figurines cultuelles égyptiennes [47]. Tout était mélangé : car les objets de cultes grecs ou anatoliens trouvés dans les ateliers de ces artisans montrent que ces derniers n'étaient pas des spécialistes de cultes exotiques [48]. On pourrait faire la même remarque pour les artisans de Pompéi : ce sont les mêmes qui sculptent les statuettes en terre cuite d'Isis, d'Antisthène ou de Pittacos, qui vont décorer les jardins des maisons [49]. Ou encore pour les artisans de Doura Europos : I'éclectisme religieux qui se manifeste dans les décors de la ville provient des mêmes ateliers [50].

Il faut donc essayer d'échapper au déterminisme de notre formation classique, ainsi qu'à son corollaire : la défense postcoloniale des savoirs « indigènes ». Les Anciens ne raisonnaient pas, ne pensaient pas en ces termes. Il ne faut pas enfin prendre trop au sérieux le discours athénien sur l'opposition
Grecs/Barbares. Ce n'est pas à l'époque hellénistique que «les Grecs » auraient découvert que les Barbares appartenaient au genre humain. On voit bien dans Eschyle que les Perses descendent de Persée, et ni Thémistocle ni Alcibiade ni Xénophon n'avaient l'impression de devenir barbares parce qu'ils fréquentaient l'aristocratie perse. Cela ne veut bien sûr pas dire que ce monde était un monde sans conflits, irénique. Mais que les savoirs « des autres » étaient beaucoup plus familiers que ce que nous penserions. C'est nous, Modernes, qui avons inventé l'idée, au siècle dernier, que l'ennemi n'était pas humain [51].

Prenez ce texte, extrait des Entretiens d'Épictète :

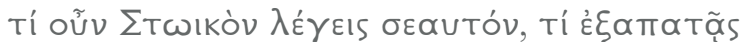

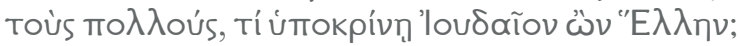

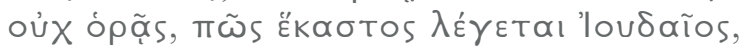

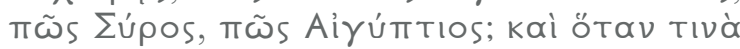

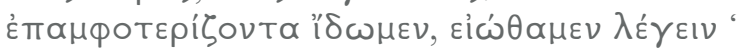

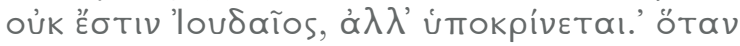

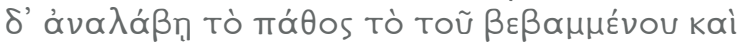

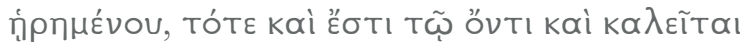

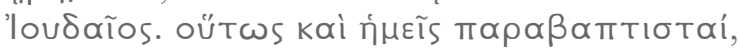

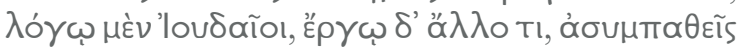

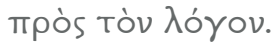

Pourquoi te dis-tu Stoïcien ? Pourquoi veux-tu tromper les gens ? Pourquoi joues-tu au Juif, alors que tu es Grec ? Est-ce que tu ne vois pas ce qui fait qu'on dit qu'un homme est juif, syrien ou égyptien ? Quand nous en voyons un louvoyer entre les deux, on dit : «Ce n'est pas un Juif : il joue le rôle d'un Juif. » En revanche, s'il se conforme aux affects de qui a été baptisé et suit le mode de vie de son école, alors il s'agit vraiment d'unJuif, et c'est à bon droit qu'on l'appelle ainsi. De même, nous aussi, nous sommes de faux baptiseurs, juifs en paroles, mais en fait tout autre chose, incapables de nous accorder à l'ordre [du monde].
[44] Hérodote, II, 159. Cf. GReaves 2002, p. 126127. Il existait des contacts entre Milet et Naucratis (GREAVES 2002, p. 84), et I'on a retrouvé des objets égyptiens dans temple d'Aphrodite et dans la « tombe du lion » (p. 89). Sur le commerce entre Milet et l'Égypte, cf. GREAVES 2002, p. 101.

[45] Plutarque, Cimon, 18, 7-8.

[46] FOWDEN 1993, p. 46-47 et p. 50.

[47] BARRETT 2011.

[48] BARRETT 2011, p. 364-365.

[49] DeSSALES 2017.

[50] BASLEZ 2008, p. 109.

[51] Les analyses de Carl Schmitt à ce propos, dans La Notion de politique, me semblent incontestablement justes. Les sociétés libérales, en mettant la guerre hors la loi, ont conduit à une déshumanisation de l'ennemi, donc à des guerres particulièrement inhumaines « Solche Kriege sind notwendigerweise besonders intensive und unmenschliche Kriege, weil sie, über das Politische hinausgehend, den Feind gleichzeitig in moralischen und anderen Kategorien herabsetzen und zum unmenschlichen Scheusal machen müssen, das nicht nur abgewehrt, sondern definitiv vernichtet werden muß, also nicht mehr nur ein in seine Grenzen zurückzuweisender Feind ist. An der Möglichkeit solcher Kriege zeigt sich aber besonders deutlich, daß der Krieg als reale Möglichkeit heute noch vorhanden ist, worauf es für die Unterscheidung von Freund und Feind und für die Erkenntnis des Politischen allein ankommt » (SCHMITT 1963, p. 37). 
Ce texte est un des plus étonnants, et un des moins commentés, que je connaisse. Parce que les spécialistes du judaïsme antique ne savent pas quoi en faire, puisqu'il appartient à un corpus qui leur est étranger, et parce que les spécialistes de la philosophie antique sont également embarrassés. Il faut ajouter que I'antijudaïsme savant du $X X^{e}$ et $d u X^{e}$ siècle a beaucoup fait pour exclure la culture juive de l'apprentissage de la culture antique. La culture juive devait être radicalement distincte de la culture classique. Les Juifs représentaient par excellence ce « religieux » dont la classe cultivée devait s'affranchir, au contact des Grecs, des Romains et, plus généralement, des peuples indo-européens [52]. Qu'est-ce donc que cette figure de Juif vient offrir au Stoïcien ? Répondre à cette question implique de sortir de nos façons de penser les savoirs antiques. À commencer par cette division en rubriques nationales. Un dernier exemple : dans son livre de référence sur le catalogue de Callimaque, Rudolf Blum dit que ce texte était la première présentation générale de la littérature nationale grecque [53]. C'est une énorme absurdité : la bibliothèque devait enfermer «les livres de tous les peuples » [54], dont la Septante (on a même repéré une citation d'Isaïe dans une épigramme de Callimaque [55]), et 800 livres attribués à Zoroastre, auprès desquels I'Iliade et I'Odyssée faisaient figure de «minuscules bréviaires», pour le dire avec Canfora [56].

\section{-ISMES}

Une autre entrave, me semble-t-il, à I'histoire des savoirs antiques, est I'habitude que nous avons de recourir à des catégories en -ismes pour désigner des courants de pensée, des courants doctrinaux : orphisme, pythagorisme, hermétisme, gnosticisme, judaïsme, christianisme. Ces -ismes donnent lieu à des débats interminables sur leurs critères (qu'est-ce qui est hermétique ? gnostique ?) et à des mises en confrontation avec des types de savoirs conçus eux aussi comme des blocs cohérents : «la » philosophie (« judaïsme et philosophie »), « la » magie (« orphisme et magie »), l'alchimie.

Je crois que, de même que les «thèmes » ne peuvent pas servir de critère pour définir des
« genres », les « doctrines » (philosophiques ou religieuses) ne peuvent pas servir de critère pour définir des « courants de pensée ». Ces derniers me semblent d'ailleurs avoir dans I'Antiquité une existence aussi incertaine que les «genres ». De même qu'il y a des « formes » ( $\left.\varepsilon^{\prime} \delta \eta\right)$ données aux livres, et qu'il ne faut pas confondre avec nos « genres», il y a des « écoles » (aipéoદıs), des associations, des groupes cultuels, mais l'unité de ces groupes vient de ce qu'on s'y réclame d'un certain fondateur (Platon, Orphée, Pythagore, le Christ, etc.) et non pas de ce qu'on y partage un même credo doctrinal. Une immense liberté était laissée à chacun d'inventer à sa guise des façons d'être platonicien, orphique, etc. À tel point qu'on remarquait quand un groupe se montrait sourcilleux en matière d'orthodoxie (comme le Jardin). Cette importance que nous accordons aux doctrines, aux convictions, aux différentes formes de « foi », vient du substrat chrétien de notre culture.

Là encore, comme à propos des distinctions par « nation », nous devons être attentifs aux confluences, aux échanges, plus qu'à des frontières peut-être inexistantes entre des blocs. Prenons par exemple la bibliothèque dite « gnostique » de Nag Hammadi. On y trouve des textes dits « hermétiques » et un texte dit «philosophique », extrait de la République de Platon. À I'intérieur même de certains textes « gnostiques », on trouve des allusions à des doctrines épicuriennes. Faut-il penser que la collection associe consciemment trois types de savoir différents, ou faut-il penser qu'elle nous dit au contraire que, du point de vue des usagers de ces textes, ces textes relèvent d'un même type de savoir ? Je pencherais pour la deuxième possibilité, d'autant plus que les catégories de « gnostique » et $d^{\prime} \ll$ hermétique » sont modernes : Michael Allen Williams a magistralement démonté la catégorie « gnostique » (ses collègues spécialistes du gnosticisme ne l'ont pas suivi), et un tel travail serait à faire pour la catégorie d'hermétisme. Un autre exemple pourrait être Stobée, qui lui aussi associe des savoirs que nous séparons en « hermétiques » et « philosophiques ».

Nous devons donc prendre pour fil d'Ariane non pas nos attentes (des groupes à cohérence doctrinale), mais ce que nous disent les acteurs euxmêmes. Ils ne pensent pas par-ismes.
[52] Je renvoie ici au magistral livre de Demoule 2015.

[53] BLUM 1991, p. 61.

[54] Tzetzes, De comoedia, p. 43 (éd. Koster).
[55] Cf. CANFORA 1990, p. 51. Callimaque, Ep., 55, 4, qui fait écho à Is., 14, 12 .

[56] CANFORA 1990, p. 32. 


\section{DISCIPLINES}

Passons maintenant à la question des disciplines, en commençant par donner une brève définition de ce mot : une discipline est un savoir qui se distingue par un objet et une méthode propres, spécifiques, par conséquent par des frontières bien délimitées avec d'autres disciplines, et enfin par des structures de reproduction des professionnels de la discipline. Y avait-il des disciplines dans I'Antiquité ? Il me semble que non, et voici pourquoi.

Nous ne devons pas confondre profession et discipline. Il y avait dans I'Antiquité des gens qui se définissaient comme philosophes, médecins, rhéteurs, etc. Mais de là à penser que ces gens appartenaient à des disciplines différentes, il y a un pas à ne pas franchir. Car très souvent, les hommes de savoir avaient des compétences communes et offraient des prestations semblables. Je prends deux exemples: nous avons I'habitude de penser qu'il existait dans I'Antiquité une distinction très importante entre «philosophie » et « rhétorique», distinction qui aurait été vécue tantôt sous forme de conflit, tantôt sous forme de complémentarité. En réalité, ce qui apparaît massivement dans nos sources, c'est que les philosophes enseignaient l'éloquence aussi bien que les rhéteurs. Lorsque les philosophes sont chassés d'Athènes en 307 av. J.-C., un fragment comique les décrit comme ceux qui transmettent aux jeunes ce qu'ils appellent les puissances des discours [57]. Fronton, qui se considère, à parts égales, comme le disciple d'Athénodote, philosophe stoïcien, et celui du rhéteur Denys [58], doit au philosophe, non au rhéteur, sa compétence en matière d'images (Eiḱkóves) [59].
Les maîtres de rhétorique devaient réciproquement avoir des compétences en matière d'éthique : comme en témoigne une inscription d'Olympie, Gorgias entraînait également ses élèves à la vertu [60], et pratiquait un certain mode de vie, ne laissant aucune place au plaisir, et c'est à ce mode de vie qu'il attribuait sa longévité [61]. Nous retrouvons cette compétence éthique chez son élève Isocrate [62], chez Diophane de Mytilène, chez Quintilien, selon qui bien souvent les philosophes sont les pires exemples en matière éthique, et jusque dans l'Antiquité tardive, par exemple chez le rhéteur Eusèbe qui résiste jusqu'à la mort au César Gallus [63], ou encore chez le rhéteur Helpidius qui initie Priscillien d'Avila à l'ascétisme [64]. Le grammairien Domitius pouvait de la même façon lancer au philosophe Favorinus que lui, grammairien, cherchait les uitae iam atque morum disciplinas, tandis que les philosophes avaient abandonné cette quête pour de vaines recherches de vocabulaire [65].

Philosophes et rhéteurs partageaient donc généralement les mêmes compétences.

Il en va de même pour les différences entre philosophes et médecins. Les différences de métiers n'empêchent pas une confluence des savoirs. Par exemple, pour décider s'il est possible qu'une grossesse dure 11 mois, Hadrien fait appel à un conseil formé de médecins et de philosophes [66]. De Galien, Marc Aurèle disait qu'il était « le premier des médecins, et le seul des philosophes qui le fût vraiment » [67]. Pourquoi ? Parce que Galien seul avait été capable de déceler la cause d'une grave indisposition alimentaire, en laquelle les médecins de la cour voyaient, à tort, le début d'une maladie. Cette confluence des savoirs entre médecins et
[57] Alexis, fr. 99 (éd. Kassel-Austin) = Athénée, XIII 610e : «Que les dieux bénissent Démétrios et les législateurs, pour avoir ordonné à ceux qui transmettent aux jeunes, comme ils disent, les puissances des discours, de quitter l'Attique et d'aller se faire pendre! ».

[58] Ad M. Caesarem et inv., II, 1, 3, p. 17 Van den Hout. [59] Ad M. Caesarem et inv., IV , 12, 2, p. 65 Van den Hout : ego, qui a meo magistro et parente Athenodoto ad exempla et imagines quasdam rerum, quas ille kikóvas appellabat, apte animo comprehendundas adcommodandasque mediocriter institutus sum [...].

[60] Ep., no 875 a (éd. Kaibel) = fr. A 8 b (éd. Diels-

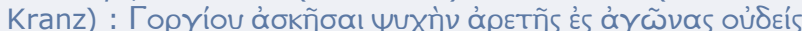

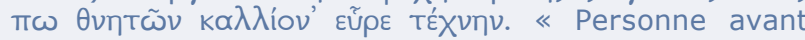
Gorgias n'avait découvert l'art d'entraîner l'âme aux joutes où I'on rivalise de valeur ».

[61] Athénée, XII, 548 c-d = fr. A 11 (éd. Diels-Kranz), où Gorgias explique sa longévité par le fait qu'il n'a

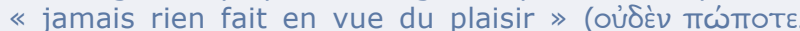

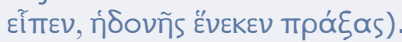

[62] Cf. Nic., 36, où les « exercices portant sur l'âme »

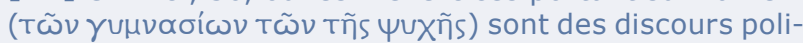
tiques, éristiques, sans nulle dimension «spirituelle ».

[63] Ammien Marcellin, XIV, 7, 17 ; 9, 5-6. Il est significatif que BROWN 1992, p. 95, qui cite pourtant la défaillance du philosophus, ne mentionne pas l'exploit du rhéteur. Un tel exemple contredirait la conception, courante chez les savants, d'une spécialisation éthique du philosophe.

[64] Cf. Sulpice Sévère (Chron., II, 46, 1), cité par SANCHEZ 2012

[65] Je commente ce face-à-face dans VESPERINI 2013.

[66] Aulu-Gelle, Nuits attiques, III, 16.

[67] Galien, Pronostic, 11, p. 128, 25-6 (éd. Nutton) :

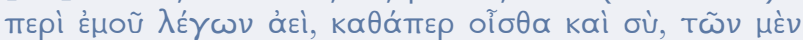

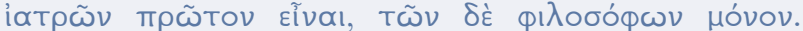
« [Marc Aurèle] disait toujours à mon propos, tu le sais, que, si j'étais le premier des médecins, j'étais le seul philosophos ». 
philosophes se retrouve d'ailleurs chez les philosophes arabes et les philosophes juifs. Elle se perd chez les chrétiens, à partir du moment où l'Église, à la fin de l'Antiquité, interdit aux prêtres d'exercer la médecine [68].

Cette confluence des savoirs s'explique aussi par la façon dont étaient conçus les savoirs lettrés. Comme une ronde, comme un cercle, où chaque savoir donnait la main aux autres. J'y reviendrai à propos des savoirs mythographiques. L'idée d'un spécialiste (de médecine, de philosophie) qui ignorerait les autres savoirs était inconcevable.

Celui qui présidait à tous ces savoirs, c'était Homère. On le voit sur le célèbre relief d'Archélaos, où les personnifications de $\mu \tilde{u} \theta$ os, d'iotopía, de moínoıs,

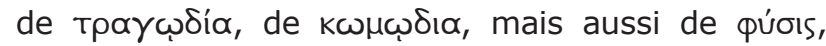
d' àpetrí, de $\mu \nu \eta ́ \mu \eta$, de míotıs et enfin de бopía, viennent sacrifier un taureau à Homère (fig.1).

Ou encore dans cette exèdre bordant une route dans le sanctuaire de Sérapis à Memphis (Saqqara), où Homère est entouré par Platon, Pindare, Protagoras et d'autres oopoí non identifiés (fig. 2).

Sur la légende de la photo, de façon caractéristique, Paul Zanker écrit « Poètes et philosophes ». Zanker projette donc sur l'image un « partage des tâches » disciplinaire, typiquement moderne. S'il y a d'un côté des poètes, de l'autre des philosophes, où classer Homère, qui figure au centre ? On ne

[68] Canon, 139, 2. peut comprendre cette exèdre que si I'on comprend que, au-delà de leurs métiers, ce sont des бopoi qui sont réunis autour du père de la oopía. Tous ont en partage le même savoir, c'est-à-dire, très concrètement, le savoir de la totalité des choses (Tà övтa en grec, res en latin), totalité sur laquelle chaque savoir est une porte d'entrée. Homère était encore appelé le «périégète », parce qu'il faisait voir, les unes après les autres, toutes les choses.

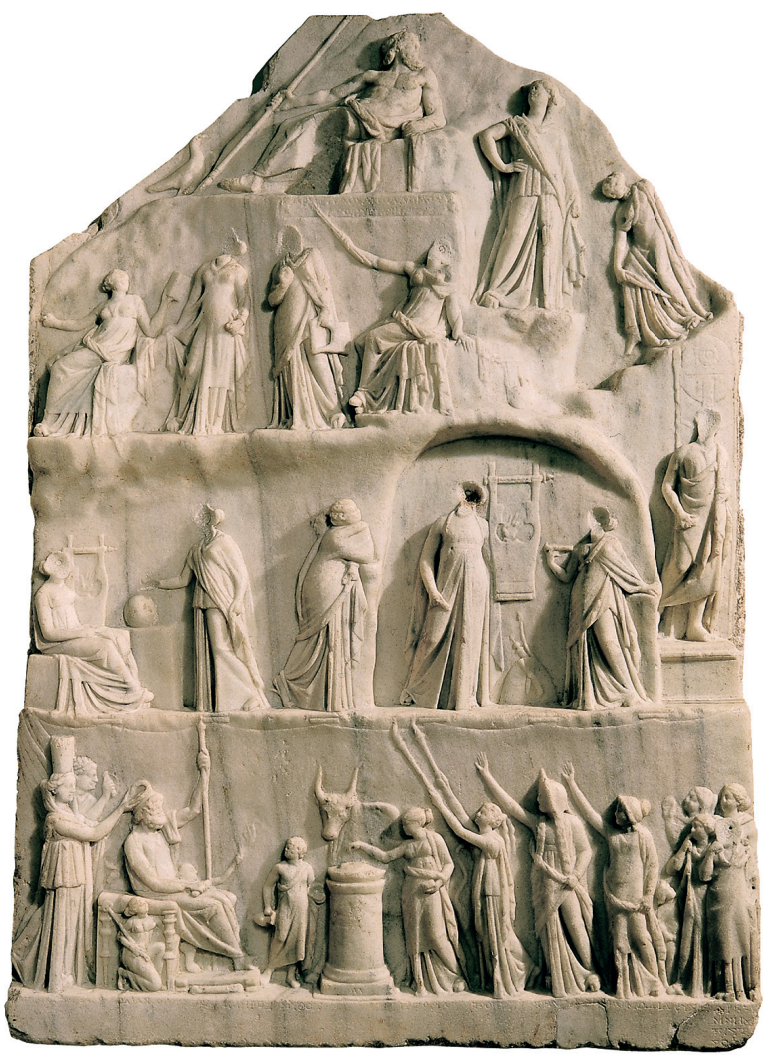

A Figure 1

Le bas-relief hellénistique d'Archélaos de Priène, British Museum.

$<$ Figure 2

Exèdre avec « sages ». Sanctuaire de Sérapis, Memphis (Saqqara). 
En un sens, chaque savoir, chaque objet de savoir, était appelé à faire accomplir une périégèse de tous les savoirs.

6. J'ai été très long sur cette cinquième ligne. C'est celle en fait qui comprend ce que Michel Foucault appelait les «synthèses toutes faites » [69], qui occupent une place centrale dans nos recherches sur les savoirs.

Je vais maintenant, à dessein, être beaucoup plus bref, et ce sera d'autant plus facile que ce qui reste apparaîtra, je pense, comme évident. Nous concevons a priori une activité de savoir comme orientée par une fin - la recherche de la vérité - et un moyen - la raison. C'est par conséquent pour nous une activité de l'âme (intellectuelle, spirituelle, intérieure), par conséquent encore une activité relevant de l'abstraction. Et, d'autre part, c'est une activité sérieuse, qui nous « engage », c'est-à-dire implique et transforme notre subjectivité, nos aspirations, nos convictions.

Cette conception du savoir a bien sûr des conséquences dans nos façons d'appréhender les savoirs antiques. Je vais en retenir trois, que je développerai inégalement. Pour commencer, nous avons tendance à négliger ou à ignorer les savoirs du corps : savoirs des danseurs, des acteurs, des entraîneurs, des athlètes, des masseurs ; et nous avons aussi tendance à négliger ou à ignorer la dimension physique des savoirs que nous qualifions d'intellectuels. Nous le faisons d'autant plus facilement que nous nous représentons ce que nous nommons « âme », « intellect», « esprit » comme quelque chose d'ontologiquement différent du corps. Dans le monde antique, on ne concevait pas ces réali-

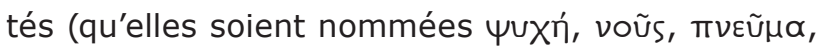
animus, mens, anima, etc.) comme des réalités d'une essence ou d'une nature différentes que le corps. C'était des réalités physiques, matérielles, et qui, comme telles, déterminaient le corps : l'âme commande le corps, dit par exemple Marc Aurèle, « comme le fil la marionnette » [70]. Le fil n'est pas d'une nature différente que la marionnette.

Dès lors, quiconque prétendait être un spécialiste du corps (médecin, entraîneur) devait également être compétent en ce qui concerne «l'âme ». Un entraîneur doit pouvoir distinguer le caractère éthique

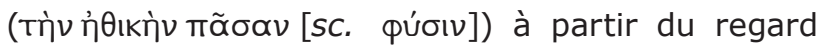
de l'athlète [71]. C'est cette compétence éthique qui

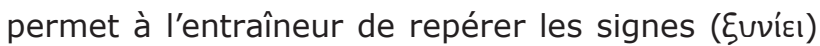
d'un náӨoc comme l'amour chez un élève [72]. Mais la réciproque était vraie : quiconque prétendait être un spécialiste de l'âme devait également être compétent en ce qui concerne le corps. Cela explique que les philosophes aient eu des compétences médicales, comme nous l'avons dit plus haut, mais aussi, plus généralement, des compétences en matière de corps : il est ainsi frappant de constater, dans la Lettre VII, que Platon était consulté pour des

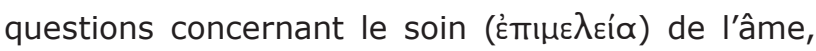
mais aussi le soin du corps - ainsi que le soin de son

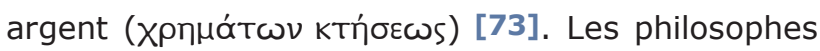
pouvaient par exemple conseiller des athlètes à la façon des entraîneurs : la oopia de Pythagore, nous dit Porphyre, permit à l'athlète Euryménès, « malgré sa petite taille, de l'emporter sur des adversaires de haute taille et d'être vainqueur à Olympie » [74].
[69] FOUCAULT 1969 , p. 32-33 : « Il faut remettre en question ces synthèses toutes faites, [...] ces liens dont la validité est reconnue d'entrée de jeu. [...] II faut aussi s'inquiéter devant ces découpages ou groupements dont nous avons acquis la familiarité. Peut-on admettre, telles quelles, la distinction des grands types de discours, ou celle des formes ou des genres qui opposent les unes aux autres science, littérature, philosophie, religion, histoire, fiction, etc., et qui en font des sortes de grandes individualités historiques? Nous ne sommes pas sûrs nous-mêmes de l'usage de ces distinctions dans le monde de discours qui est le nôtre. À plus forte raison lorsqu'il s'agit d'analyser des ensembles d'énoncés qui étaient, à l'époque de leur formulation, distribués, répartis et caractérisés d'une tout autre manière : après tout, la "littérature" et la "politique" sont des catégories récentes qu'on ne peut appliquer à la culture classique que par une hypothèse rétrospective, et par un jeu d'analogies formelles ou de ressemblances sémantiques; mais ni la littérature, ni la politique, ni non plus la philosophie et les sciences n'articulaient le champ du discours, au XVII ${ }^{\mathrm{e}}$ ou au XVIII ${ }^{\mathrm{e}}$ siècle comme elles l'ont articulé au $X I X^{e}$ siècle. »
[70] Marc Aurèle, X, 38 ; XII, 19.

[71] Philostrate, Sur la gymnastique, 25.

[72] Philostrate, Sur la gymnastique, 22. Cf. aussi les exhortations et les invectives que les entraîneurs adressent à leurs élèves (Philostrate, Sur la gymnastique, 20

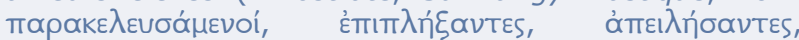
бoфıoáuєvoı) sont les mêmes que celles adressées par Blossius à Tiberius Gracchus, par exemple. Cf. les exemples donnés par Philostrate, Sur la gymnastique, 21-24 et 53.

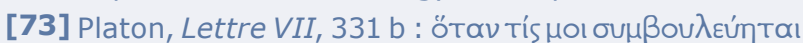

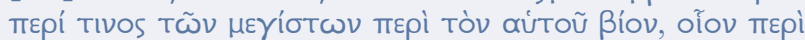

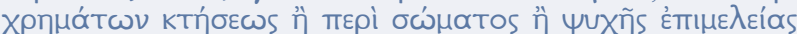
[...]. « Quand quelqu'un me consulte sur un des points les plus importants de son existence, par exemple l'acquisition de biens, ou le soin du corps ou de l'âme [...] ».

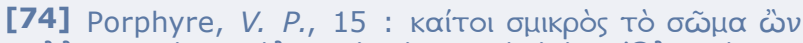

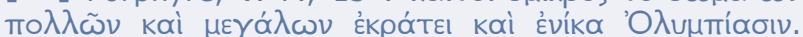
La ooфía de Pythagore consiste en l'occurrence à avoir conseillé un régime à base de viande, plutôt de que de fromage et de figues. Cf. aussi Diogène Laërce, VIII, 12-13. Sur l'importance d'un régime alimentaire « solide » (on pratiquait même l'ávałkoqayía) pour réussir à la lutte, cf. DECKER \& THUILLIER 2004, p. 99. 
Certains d'entre eux pouvaient être entraîneurs : on a par exemple retrouvé l'épitaphe d'un certain Philocratès de Sidon, venu de Syrie entraîner les jeunes citoyens d'Orchomène, qui semble avoir été en même temps un philosophe épicurien [75]. Il est caractéristique que dans la notice consacrée à ce Philocratès dans le Dictionnaire des philosophes antiques, Bernadette Puech propose une interprétation « métaphorique » de son activité d'entraîneur. Bien souvent, quand quelque chose nous étonne, nous avons la tentation de l'explain away, comme disent les Anglais, en y voyant une métaphore. Cette lecture métaphorique est une sorte de lectio facilior, dont il faut par conséquent se méfier. En l'occurrence, rien ne s'oppose à ce qu'un entraîneur se soit aussi présenté comme un Épicurien. De nombreux philosophes furent de grands athlètes : sans parler de Milon, six fois vainqueur à la lutte aux jeux olympiques [76], et sans remonter à Empédocle [77] et à Platon [78], signalons que I'Académicien Chairon de Pellène fut « deux ou trois fois vainqueur à la lutte, sans jamais tomber »[79], qu'Antisthène était lutteur [80] et que Lycon, scolarque du Lycée [81], fut au départ pugiliste (i. e. boxeur), tout comme le Stoïcien Cléanthe [82]. Sous I'Empire, Sostratos et Démonax sont de vrais Hercules, qui construisent des ponts et tuent les brigands [83]. Qu'ils aient eu des compétences de médecin, d'entraîneur, d'athlète, l'important est de retenir que des spécialistes de l'âme devaient en même temps être des spécialistes du corps, et cela devait se voir avant tout à leur corps : «On se retournait dans la rue pour regarder le corps de Diogène », dira Épictète [84], tandis que pour nous, au contraire, un intellectuel ne doit pas nécessairement avoir un corps d'athlète.

[75] IG VII 3226 = GG 201.

[76] Diodore de Sicile, XII, 9, 6, qui ajoute que lors de la bataille décisive entre Crotone et Sybaris, Milon, qui commandait les Crotoniates, combattit vêtu en Héraclès. [77] Diogène Laërce, VIII, 53.

[78] Diogène Laërce, III, 4 : son surnom de Platon (« le costaud, le large ») lui aurait été donné par son pédotribe, le lutteur Ariston, et il aurait participé aux concours isthmiques comme lutteur. Sur la silhouette des lutteurs, «très lourds et corpulents », cf. DECKER \& THUILLIER 2004, p. 98-99 et pl. XII et XIV.

[79] Acad. Hist., col. XI Dorandi. Cf. DPhA (C 92). Pour être vainqueur, il fallait projeter trois fois son adversaire à terre. Donc Chairon était toujours vainqueur par des scores de 3-0 et non, comme généralement, par 3-2 ou 3-1. Cf. DeCKer \& Thuillier 2004, p. 98.

[80] Diogène Laërce, VI, 4.
Nous devons donc être attentifs, d'une part, à ne pas négliger, parmi les savoirs, les savoirs physiques. Et à ne pas négliger non plus l'aspect matériel, physique, corporel, de tous les savoirs. On oppose ainsi couramment les écrits « hermétiques » entre « écrits techniques » et « écrits philosophiques », alors que de nombreux écrits her-

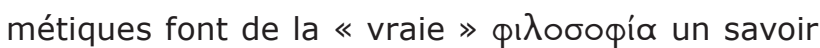
technique, opératoire, et alors que, bien souvent, les textes que nous croyons «philosophiques » car «non-techniques » ont été en fait expurgés à époque byzantine [85].

Cette tendance à concevoir le monde des savoirs comme un monde «idéal », «pur », immatériel, désincarné, s'explique par la même raison qui faisait que Lavoisier avait honte de se dire chimiste, et préférait se dire physicien [86]. Je suis convaincu qu'il s'agit là d'un héritage du christianisme, héritage sécularisé bien sûr. Mais qui n'en a pas moins maintenu I'opposition, établie par les Pères de I'Église, entre «la lettre » et «l'esprit», entre sensus carnalis et sensus mysticus. Les savants d'aujourd'hui doivent être particulièrement vigilants face à cette « spiritualisation » de leur regard sur les savoirs antiques, d'autant plus forte qu'elle est souvent inconsciente. Ils doivent au contraire, comme les savants juifs critiqués par les disciples d'Origène, devenir un carnalis populus [87].

J'en viens maintenant à I'opposition entre les savoirs et ce qui relève pour nous de I'« imagination »: fables, récits, mythes. Pour nous, il ne saurait y avoir rien de commun entre l'astronomie et la mythologie. L'une relève de la science, de la recherche de la vérité, l'autre de la « littérature », de la fiction. On est donc très étonné de lire, au début de la Bibliothèque d'Apollodore, cette épigramme, que Photius a transcrite:

[81] Diogène Laërce, $v, 67$

[82] Diogène Laërce VII, 168.

[83] Lucien, Dem, 1.

[84] III, 22, 88.

[85] FOWDEN 1993, p. 117-118.

[86] Cf. LASZLO 2000, p. 243, qui parle de « syndrome de Lavoisier ». Je dois cette référence à BouRDIEU 2001, p. 132 , dont je voudrais reproduire le commentaire si éclairant : «Science descriptive et empirique, occupée à des tâches pratiques et appliquées (engrais, médicaments, verre, insecticides) et utilisant des recettes (d'où I'analogie avec la cuisine), la chimie est toujours décrite comme une servante ».

[87] RoSENTHAL 1969, p. 256 : « Already the Church Fathers opposed to the sensus Judaicus (by which they mean the literal sense) the sensus mysticus. For the disciples of Origen the Jews were a carnalis populus, amici litterae ». 


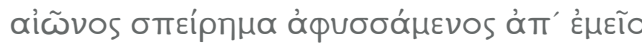

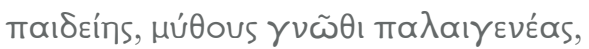

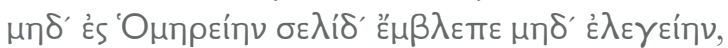

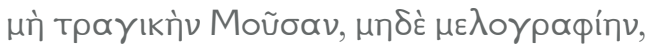

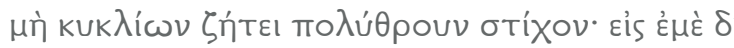
$\dot{\alpha} \theta \rho \tilde{\omega} v$

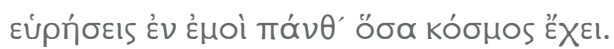

\author{
De moi tu tires la semence de l'éternité : \\ la connaissance des récits antiques. \\ Ne regarde pas Homère ni l'élégie, \\ ni la Muse tragique, ni la mélique, \\ ne va pas chercher les vers sonores des poèmes \\ cycliques : regarde-moi, \\ en moi tu trouveras tout ce que contient I'univers.
}

La mythographie faisait partie du monde des savoirs lettrés. J'ai dit plus haut que l'une des raisons pour lesquelles l'idée de « discipline » posait problème, c'est que les savoirs étaient conçus comme un cercle à l'intérieur duquel chaque «savoir » (poésie, histoire, philosophie...) constituait un point de départ vers tous les autres savoirs. Il en va de même pour la mythologie. Comment la mythologie peut-elle prétendre renfermer «tout ce que contient l'univers »? Si nous lisons le commentaire de Photius, une première réponse est donnée : apprendre la mythologie, c'est apprendre tous les lieux géographiques du monde. On voit ici combien nos modes de lecture sont éloignés des Anciens : pour nous, les noms géographiques qui scandent les récits mythologiques sont secondaires, accessoires. Nous n'irions jamais penser qu'ils permettaient d'apprendre tout un inventaire du monde. À partir de l'apprentissage des noms géographiques, on pouvait apprendre les différentes histoires liées à chacun de ces lieux : les dynasties qui y avaient régné, les

[88] Servius, ad Aen. 6, 445 : nunc femina Caenevs. Caenis virgo fuit, quae a Neptuno pro stupri praemio meruit sexus mutationem. Fuit etiam invulnerabilis. Qui pugnando pro Lapithis contra Centauros crebris ictibus fustium paulatim fixus in terra est, post mortem tamen in sexum rediit. Hoc autem dicto ostendit Platonicum illud vel Aristotelicum, animas per $\mu \varepsilon т \varepsilon \mu \psi u \dot{x} \omega \sigma \mathrm{IV}$ sexum plerumque mutare.

[89] Platon, Phèdre, 276b.

[90] Antigone de Carystos, fr. 23 (éd. Dorandi) = Athénée, XII, 547f-548a. «Platon, Speusippe et leurs disciples instituèrent ces réunions afin de montrer qu'ils rendaient un culte aux dieux et qu'ils se comportaient les uns avec les autres en conformité avec la Nature, mais surtout, parce qu'ils visaient la détente et le plaisir des discours savants ». grands hommes qui y étaient nés, les monuments qu'on y montrait, les rites qui s'y pratiquaient, les phénomènes étonnants qui $s^{\prime} y$ produisaient, les plantes et les animaux dignes de mémoire, etc. La mythologie pouvait encore donner accès à l'apprentissage des doctrines philosophiques. Par exemple, à partir de deux vers sur Cénée (une princesse lapithe violée par Poséidon, qui lui accorde ensuite le vœu d'être un homme, et que Virgile fait défiler aux Enfers, nunc femina rursus, parmi les femmes malheureuses en amour), Servius explique que Virgile a voulu illustrer cette idée platonicienne aussi bien qu'aristotélicienne, selon laquelle en général, par la métempsychose, les âmes changent de sexe [88]. Ce commentaire de Servius est aujourd'hui le fr. 12 (éd. Ross) de I'Eudème d'Aristote. On pourrait multiplier les exemples.

J'en viens maintenant à mon dernier point, qui est peut-être le plus difficile à comprendre et à communiquer. Pour nous, une activité de savoir est donc orientée par la recherche de la vérité, et cette recherche de la vérité n'est pas quelque chose de distant par rapport à nous : cette recherche est censée engager toute une intériorité, plus ou moins tourmentée (Faust constitue ici un paradigme essentiel du savant contemporain). Cette recherche engagée de la vérité est, entre autres, ce qui fait, à nos yeux, la noblesse du métier de savant. Or bien souvent, les savoirs lettrés antiques nous confrontent à des pratiques qui ne sont pas orientées par la recherche de la vérité, mais par la recherche du plaisir en commun, plaisir qui provient de la coexistence, de la multiplication, de la prolifération de vérités variées. Cette recherche du plaisir n'engage pas des «intériorités», mais des spectateurs et des auditeurs passionnés par le déploiement du savoir, conçu comme un événement esthétique. Le savoir se donne alors comme une surface, et non comme une profondeur.

En un mot, les savoirs lettrés font très souvent I'objet d'une pragmatique ludique. Or il n'y a rien de plus difficile à concevoir pour nous qu'un jeu qui se joue autour du savoir, qu'un savoir qui s'expérimente essentiellement dans le jeu. Mais c'est bien ainsi que les savoirs se présentent à nous dans les riches demeures athéniennes du siècle de Périclès, dans les dialogues où Platon a semé des

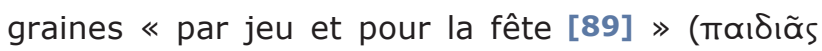

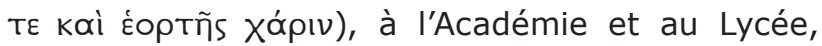
fondées, nous dit Antigone de Carystos, en vue de la détente des entretiens savants [90], dans les 
travaux des savants du Musée, comme l'a magnifiquement montré William Slater [91] dans un article trop peu connu, dans les conversations savantes que nous font connaître Plutarque, Athénée, Lucien, Macrobe et alii, et jusque dans les recherches, pour nous on ne peut plus « sérieuses », $d$ 'Ératosthène ou d'Archimède [92].

Prenons par exemple les Moralia de Plutarque : ces dialogues sont-ils à lire comme des textes sérieux, des traités de philosophie dans lesquels Plutarque exposerait ses idées ? C'est ainsi qu'ils sont lus couramment. Cette interprétation me paraît souvent discutable, notamment lorsqu'elle fait de tel ou tel personnage un « double » ou au contraire une « cible » de Plutarque [93], sans qu'aucun indice ne vienne appuyer ces interprétations, ou encore lorsqu'elle prétend classer les Moralia par « disciplines »: le De Pythiae oraculis, avec ses discours sur l'origine du bronze corinthien ou les épingles de Phryné, est-il un dialogue «théologique » [94] ? On peut donc se demander si les Moralia ne fonctionnaient pas différemment d'un traité.

Le monde antique des savoirs lettrés se présente donc très souvent à nous comme un monde de la

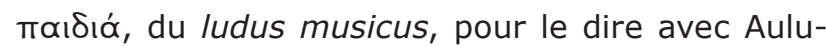
Gelle. Et la condamnation du jeu par l'Église [95], ainsi que l'injonction qui nous est faite de travailler toujours plus, ne nous ont pas préparés à distinguer, encore moins à valoriser, cette dimension pourtant essentielle. Une autre difficulté tient au fait que le phénomène du jeu, d'une manière générale, est notoirement difficile à définir. Il faut là aussi partir de ce que nous disent nos « informateurs » antiques. Alors que pour nous, le jeu est un « divertissement», opposé au sérieux de la vie professionnelle et au sérieux de la vie intellectuelle, pour les Anciens, le jeu, comme la fête, est une façon d'imiter les dieux [96] ; c'est encore le fondement de la vie sociale; et c'est enfin la marque d'une vie libre et digne, car exempte du móvos.

La christianisation du monde antique a mis fin, non pas tant à la dimension ludique des pratiques de savoir, mais à la valorisation de cette dimension ludique. L'Église va progressivement imposer l'idée que : 1) l'accès à la Vérité n'est pas une initiation à une vision incommunicable, mais l'intériorisation d'une doctrine, donc d'un discours, sous forme de « foi »; 2) cette doctrine est la seule vraie ; 3) ce ne sont pas en dernière analyse les actions justes, mais la foi qui permet d'être sauvé [97] ; 4) le salut de l'État et du monde est suspendu à l'issue de la lutte entre la Vérité et les «fausses doctrines». Par conséquent, tous les hommes ont le devoir de s'initier à cette vérité, et le devoir de l'État est de conduire les hommes, par la persuasion ou par la violence, sur l'iter salutare [98] qui mène à la Vérité.

Dans ces conditions, on comprend que les jeux de savoir étaient condamnés. Je voudrais citer un seul texte, de saint Augustin [99], car il est magnifique dans la vivacité, où l'amour le dispute à la haine, avec laquelle il décrit, tout en lui donnant congé, ce qui était pour lui «le monde d'hier »:

\section{Et hoc erat ingens fabula et longum mendacium, cuius adulterina confricatione corrumpeba- tur mens nostra pruriens in auribus. sed illa mihi fabula non moriebatur, si quis amicorum}

[91] SLATER 1982.

[92] Cf. le grand livre, lui aussi trop peu connu, de NETZ 2009.

[93] Sarapion est présenté par PUECH 1992, p. 4875 comme « un moraliste austère, défenseur inconditionnel de la religion [...], un personnage grave et solennel, d'une respectabilité décourageante $»$, envers lequel Plutarque aurait une « admiration imperceptiblement teintée d'ironie ». Il s'agit d'une appréciation qui n'est fondée que sur l'idée qu'il doit y avoir dans les dialogues de Plutarque des « adversaires » des positions philosophiques de leur auteur. En réalité, absolument rien, dans les textes de Plutarque, ne permet de fonder cette description de Sarapion.

[94] Je fais référence ici au classement des Moralia proposé par Konrat Ziegler, qui fait encore aujourd'hui autorité. J'ai analysé le fonctionnement de ce dialogue dans VESPERINI 2015a.

[95] Cf. HAMAYON 2012 (chapitre 2).

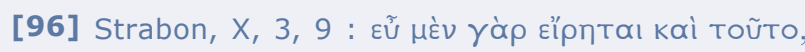

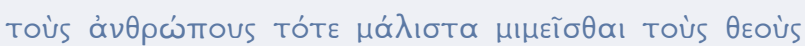

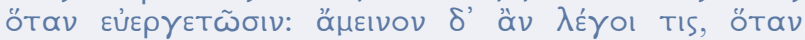

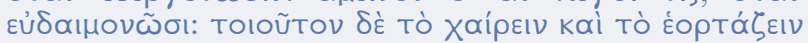
kaì Tò фı les hommes imitent les dieux surtout quand ils dispensent des bienfaits, et c'est bien dit. Mais on aurait encore plus raison de dire : quand ils sont heureux. Or ils accèdent à cet état quand ils sont en joie, quand ils font la fête, quand ils font de la philosophia, et quand ils musiquent ».

[97] Augustin, Contra duas epistulas pelagianorum, 3, 5, 14 (BA 23, p. 502 s.) : un homme parfaitement juste mais ayant une foi branlante ne sera pas sauvé, tandis que celui qui a une foi parfaite sera sauvé, même s'il n'est pas d'une vertu parfaite, par exemple s'il se met parfois en colère ou fait l'amour avec sa femme pour le plaisir et non pour la procréation. C'est tout l'enjeu de la lutte contre Pélage et Julien d'Éclane.

[98] Lettre au concile d'Arles (p. 78 dans le recueil de V. Keil), citée par VEYNE 2010, p. 20 et 83.

[99] Saint Augustin, Confessions, IV, 8. 
meorum moreretur. alia erant quae in eis amplius capiebant animum, conloqui et conridere et uicissim beniuole obsequi, simul legere libros dulciloquos, simul nugari et simul honestari, dissentire interdum sine odio tamquam ipse homo secum atque ipsa rarissima dissensione condire consensiones plurimas, docere aliquid inuicem aut discere $a b$ inuicem, desiderare absentes cum molestia, suscipere uenientes cum laetitia: his atque huius modi signis a corde amantium et redamantium procedentibus per os, per linguam, per oculos et mille motus gratissimos, quasi fomitibus conflare animos et ex pluribus unum facere.

Énorme fable, long mensonge qui corrompait mon esprit : parler ensemble, rire ensemble, échanger des services avec bienveillance, lire ensemble des livres au langage séduisant, plaisanter ensemble, échanger des marques d'honneur, exprimer parfois un désaccord, mais sans haine, comme on le fait avec soi-même, et de telle sorte que le dissentiment, rarissime, relève l'unanimité qui a lieu la plupart du temps, enseigner ou apprendre à tour de rôle, regretter les absents avec peine, accueillir les arrivants dans la joie : par ces témoignages d'amour mutuel que rendaient la langue, les yeux, et mille émotions délicieuses, faire fusionner les âmes comme au moyen de combustibles, et n'en faire plus qu'une.
Il faut là encore déchristianiser (encore une fois déchristianiser une version sécularisée du christianisme) notre rapport aux savoirs antiques, en nous aidant des différentes réflexions qui ont pris le jeu pour objet. Parmi celles-ci, aucune ne me paraît plus féconde pour une anthropologie des savoirs antiques que celle de Donald Winnicott [100].

\section{CONCLUSION}

J'ai voulu, avec toute la contingence et I'arbitraire qui caractérise un travail individuel et empirique, exposer les difficultés auxquelles j'avais été confronté, en travaillant sur un objet dénommé «philosophie antique ». Je sais que cet exposé est très incomplet, mais j'ai préféré, même à ce stade balbutiant, faire part de ces préoccupations, dans l'espoir de faire gagner du temps à ceux qui souhaitent étudier les savoirs antiques. Les catégories qui ordonnent depuis deux siècles notre compréhension du savoir et des savoirs entravent notre perception des savoirs antiques, comme les grilles de la cage de la panthère dans le célèbre poème de Rilke [101]. C'est seulement en nous mettant à l'écoute des Anciens, à la façon dont les anthropologues le font avec leurs informateurs, que nous pourrons faire le mieux notre travail d'historien.
[100] Je pense bien sûr à son grand livre Play and Reality, mais aussi à un petit texte sur la reine d'Angleterre, qui me paraît également éclairer la pragmatique ludique des savoirs antiques: «Ceux qui ne voient [dans la monarchie] qu'un conte de fée, un ballet ou une pièce de théâtre - alors que c'est bien un aspect de la vie elle-même qu'ils ont sous les yeux - pourraient la tuer à force de la ridiculiser. Cet aspect de la vie demande à être soigneusement expliqué [...]. Nous sommes là dans la zone intermédiaire entre le sommeil et la veille. C'est l'espace du jeu et de l'expérience culturelle, [...] tous signes qui attestent la bonne santé psychiatrique du sujet. » Alors qu'on décrit la vie humaine selon I'alternative 《 rêve personnel - réalité vraie ou partagée », «on se rend compte que la vie des adultes, des adolescents, des enfants et des nourrissons se déroule essentiellement dans cet espace intermédiaire. On pourrait même dire que toute la civilisation se passe là »(WINNICOTT 2004, p. 388).
[101] J'emprunte cette image aux magnifiques mémoires de Woolf 1972, p. 13 : «In writing this third volume of my autobiography I have often been haunted by the lines from Rilke's superb poem "Der Panther" which I have had printed at the beginning of this book. This volume is the record of my life during the first great war and all through the war one felt that one was behind bars, and now recalling those years it seems to me that one was looking at the world and one's own life through bars. But then another thought, a terrible doubt, came to me. There are other bars, permanent bars of the cage of one's life, through which one has always and will always gaze at the world. The bars of one's birth and family and ancestors, of one's school and college, of one's own secret and sinuous psychology. Has not my mind, my soul, if I have a soul, for the last 82 years been pacing up and down like the panther, backwards and forwards, behind these bars and gazing through them until, so weary, I have seen, not the world or life, but only the bars - a thousand bars and behind the thousand bars no world? ». Je souligne. 
BARRETT, Caitlín E., 2011, Egyptianizing Figurines from Delos: A Study in Hellenistic Religion, Leiden.

BASLeZ, Marie-Françoise, 2008, Comment notre monde est devenu chrétien, Paris.

BLOCH, Marc, 1961, Apologie pour l'Histoire ou Métier d'historien (1're éd. 1949), Paris.

BLUM, Rudolf, 1991, Kallimachos: The Alexandrian Library and the Origins of Bibliography, Madison.

Bourdieu, Pierre, 2001, Science de la science et réflexivité, Paris.

Bourdieu, Pierre, 1987, Choses dites, Paris.

Bouveresse, Jacques, 2012, «Bourdieu, Pascal, la philosophie et la critique de I"illusion scolastique" », dans À temps et à contretemps, Paris (en ligne : http://books.openedition.org/cdf/2040).

BoyancÉ, Pierre, 1972, Le Culte des Muses chez les philosophes grecs. Études d'histoire et de psychologie religieuses,

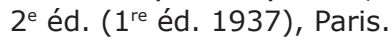

BROWN, Peter, 1992, Power and Persuasion in Late Antiquity, Madison.

BURKERT, Walter, 1991, Antike Mysterien: Funktionen und Gehalt, München.

BYL, Simon, 1997, «Controverses antiques autour de la dissection et de la vivisection », Revue belge de Philologie et d'Histoire 75, 1, p. 113-120.

CanforA, Luciano, 1990, La biblioteca scomparsa, $2^{\text {e }}$ éd. (1 $1^{\text {re }}$ éd. 1986), Palermo.

CuvignY, Marcel, 1968, «Plutarque et Épictète », dans Actes du VIIIe congrès de l'Association Guillaume Budé, Paris, p. 560-566.

DeCKer, Wolfgang \& Thurllier, Jean-Pierre, 2004, Le Sport dans l'Antiquité. Égypte, Grèce, Rome, Paris.

Demoule, Jean-Paul, 2014, Mais où sont passés les Indo-Européens ? Le mythe d'origine de l'Occident, Paris.

Dessales, Hélène, 2017, « Des philosophes domestiques ? Production statuaire et paysages culturels dans I'habitat de Pompéi », dans Pierre Vesperini (éd.), Philosophari. Usages romains des savoirs grecs sous la République et sous I'Empire, Paris, p. 487-513.

Festugière, André-Jean, 1968, Épicure et ses dieux, 2e éd. (1 $1^{\text {re }}$ éd. 1946), Paris.

FoucAult, Michel, 1969, L'Archéologie du savoir, Paris.

Foucault, Michel, 1966, Les mots et les choses, Paris.

FowDEN, Garth, 1993, The Egyptian Hermes: A historical approach to the late Pagan mind, Princeton.

GeERTZ, Clifford, 1974, «From the Native's Point of View: On the Nature of Anthropological Understanding », Bulletin of the American Academy of Arts and Sciences 28, 1, p. 26-45.

Greaves, Alan M., 2002, Miletos: A history, London - New York.

HADOT, Pierre, 2010, Wittgenstein et les limites du langage, Paris.

HADOt, Pierre, 2003, La Philosophie comme manière de vivre. Entretiens avec Jeannie Carlier et Arnold I. Davidson,

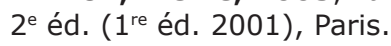

HAHN, Johannes, 1989, Der Philosoph und die Gesellschaft: Selbstverständnis, öffentliches Auftreten und populäre Erwartungen in der hohen Kaiserzeit, Stuttgart.

HAMAYON, Roberte, 2012, Jouer. Étude anthropologique à partir d'exemples sibériens, Paris.

Koch Piettre, Renée, 2005, Comment peut-on être dieu? La secte d'Épicure, Paris.

KoRHONen, Tua, 2007, «Self-Concept and Public Image of Philosophers and Philosophical Schools at the Beginning of the Hellenistic Age », dans Jaakko Frösén (éd.), Early Hellenistic Athens: Symptoms of a Change, Helsinki, p. 33-101. Kowalzig, Barbara \& WiLson, Peter (éd.), 2013, Dithyramb in Context, Oxford.

LANG, Jörn, 2012, Mit Wissen geschmückt? Zur bildlichen Rezeption griechischer Dichter und Denker in der römischen Lebenswelt, Wiesbaden.

LASzLo, Pierre, 2000, Miroir de la chimie, Paris.

Netz, Reviel, 2009, Ludic Proof. Greek Mathematics and the Alexandrian Aesthetic, Cambridge.

Petrt, Thierry, 2011, Edipe et le Chérubin. Les sphinx levantins, cypriotes et grecs comme gardiens d'Immortalité, Fribourg.

PuccI, Giuseppe, 2014, «Immagine », dans Maurizio Bettini \& William M. Short (éd.), Con i Romani. Un'antropologia della cultura antica, Bologna, p. 353-378.

Puech, Bernadette, 2005, s. v. Nigros de Chéronée, Dictionnaire des philosophes antiques IV, Paris, p. 717.

PueCH, Bernadette, 1992, « Prosopographie des amis de Plutarque », Aufstieg und Niedergang der römischen Welt II, 33.6, Berlin, p. 4831-4893.

RosenthaL, Erwin I. J., 1969, «The Study of the Bible in Medieval Judaism », dans Geoffrey W. H. Lampe (éd.), The Cambridge History of the Bible. Vol. 2: The West from the Fathers to the Reformation, Cambridge, p. 252-279.

SANCheZ, Sylvain J. G., 2012, S. v. Priscillianus, Dictionnaire des philosophes antiques Vb, Paris, p. 1522-1528.

SCheler, Max, 1926, Die Wissensformen und die Gesellschaft, Probleme einer Soziologie des Wissens, Leipzig.

Schmitt, Carl, 1963, Der Begriff des Politischen (1' éd. 1932), Berlin.

SLATER, William J., 1982, « Aristophanes of Byzantium and Problem-Solving in the Museum », Classical Quarterly 32 , p. 336-349. 
Tran, Nicolas, 2013, Dominus tabernae. Le statut de travail des artisans et des commerçants de l'Occident romain ( $I^{\text {er }}$ siècle av. J.-C. - III $I^{e}$ siècle ap. J.-C.), Roma.

VesperinI, P., «La culture antique était-elle une "culture de la transmission" ? Façons grecques et façons romaines de faire passer les savoirs », Asdiwal 12, 2017 b, p. 113-134.

VesperinI, P., Lucrèce. Archéologie d'un classique européen, Fayard, Paris, 2017 c.

Vesperini, Pierre, 2017, «De Thalès à Anaxagore : les Ioniens à l'école des dieux », Kernos (30, 2017, p. 5-32).

VesperinI, Pierre, 2015a, « Au-delà du sens. Les logoi philosophoi face aux images », Mètis 13, p. 123-150.

VesperinI, Pierre, 2015b, « La poésie didactique. Une invention des Modernes », Anabases 21, p. 25-38.

VesperinI, Pierre, 2013, « Marc Aurèle, la "vérité héroïque" et la mélancolie », Revue de philologie 87, 1, p. 153-168.

VeSPERINI, Pierre, 2010, «La categoria di "programma ideologico" è pertinente per interpretare i monumenti romani? Appunti sulla villa dei Papiri e l'ipogeo di Porta Maggiore », communication à la British School, Rome (disponible en ligne sur academia.edu).

VeYne, Paul, 2010, Quand notre monde est devenu chrétien (312-394), 2e éd. (1 éd. 2007), Paris.

Wrlliams, Michael A., 1996, Rethinking Gnosticism. An argument for dismantling a dubious category, Princeton.

WInnICotT, Donald W., 2004, Conversations ordinaires, Paris.

Wittgenstein, Ludwig, 1953, Philosophische Untersuchungen, London.

Woolf, Leonard, 1972, Beginning Again. An Autobiography of the years 1911-1918, London.

ZaNker, Paul, 1995, Die Maske des Sokrates: Das Bild des Intellektuellen in der antiken Kunst, München. 